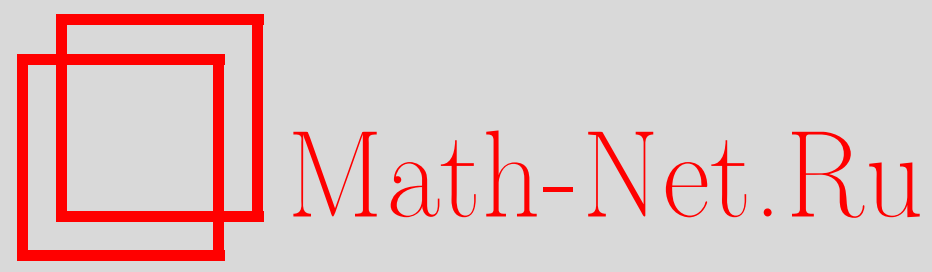

А. Г. Карапетян, О минимуме модуля тригонометрических полиномов со случайными коэффициентами, Матем. заметки, 1997, том 61, выпуск 3, 451-455

DOI: https://doi.org/10.4213/mzm1521

Использование Общероссийского математического портала Math-Net.Ru подразумевает, что вы прочитали и согласны с пользовательским соглашением http://www.mathnet.ru/rus/agreement

Параметры загрузки:

IP : 54.166 .219 .16

26 апреля 2023 г., 18:00:38 


\section{О МИНИМУМЕ МОДУЛЯ ТРИГОНОМЕТРИЧЕСКИХ ПОЛИНОМОВ СО СЛУЧАЙНЫМИ КОЭФФИЦИЕНТАМИ}

\section{А. Г. Карапетян}

Для независимых случайных величин $\xi_{0}, \ldots, \xi_{n-1}$, каждая из которых равна +1 или -1 с вероятностью $1 / 2$, обозначим через $P(u)$ выражение

$$
P(u)=P_{n}(u)=\operatorname{Pr}\left(\min _{x \in T}\left|\sum_{j=0}^{n-1} \xi_{j} \exp (i j x)\right|>u\right) \quad(u \geqslant 0) .
$$

Дж. Литтлвуд [1] предположил, что $P(\varepsilon \sqrt{n}) \rightarrow 0$ при $n \rightarrow \infty$ для любого $\varepsilon>0$. Б. С. Кашин [2] доказал эту гипотезу, установив, что $P\left(n^{1 / 2}(\log n)^{-1 / 3}\right) \rightarrow 0$ при $n \rightarrow \infty$. А. М. Одлыжко показал, что $P\left(n^{1 / 3+\varepsilon}\right) \rightarrow 0$ при любом $\varepsilon>0$ и $n \rightarrow \infty$ и предположил, что при больших $n$ и всяком $\varepsilon>0$ для большинства полиномов

$$
T(x)=\sum_{j=0}^{n-1} \pm \exp (i j x)
$$

выполнена оценка

$$
\min _{x \in T}|T(x)|<n^{-1 / 2+\varepsilon} .
$$

Эта гипотеза была доказана С. В. Конягиным [3], установившим, что $P\left(n^{-1 / 2+\varepsilon}\right) \rightarrow 0$ для любого $\varepsilon>0$ и при $n \rightarrow \infty$.

В работе мы также рассматриваем полиномы типа

$$
T(x)=\sum_{j=0}^{n-1} \xi_{j} \exp (i j x),
$$

но уже для независимых одинаково распределенных случайных величин $\xi_{j}, j=0, \ldots$, $n-1$, с конечньми третьими моментами и с нулевыми средними. Для таких полиномов, считая, что второй момент случайных величин $\xi_{j}, j=0, \ldots, n-1$, не равен 0 (случай равенства 0 второго момента случайных величин $\xi_{j}, j=0, \ldots, n-1$, является тривиальньг), доказьваем теорему.

ТЕорема. Для любого $\varepsilon \in(0,1)$ nрu $n>\left(16 C_{0}(\xi)\right)^{9936 / \varepsilon^{3}}$

$$
P\left(n^{-1 / 2+\varepsilon}\right) \leqslant \frac{1}{n^{\varepsilon^{2} / 180}},
$$

әде $C_{0}(\xi)=8\left(1 / \gamma_{0}+B+1 / A+6 \mathrm{M}|\xi|^{3}+1 /\left(\mathrm{M} \xi^{2}\right)\right), \mathrm{M} \xi^{2}-$ второй момент, $\mathrm{M}|\xi|^{3}-$ третий абсолютный момент случайной величины $\xi_{j}, j=0, \ldots, n-1, a A, B, \gamma_{0}-$ положстельные числа, удовлетворяющие соотношению

$$
\operatorname{Pr}\left(A<\xi_{1}-\xi_{2}<B\right)=\gamma_{0} .
$$

Работа выполнена при поддержке Российского фонда фундаментальных исследований, грант № 96-01-00378. 
СлЕДСТВИЕ. ДЛя $n>\left(16 C_{0}(\xi)\right)^{9936}$

$$
P\left(n^{-1 / 2+21.5\left(\ln \left(16 C_{0}(\xi)\right) / \ln n\right)^{1 / 3}}\right) \leqslant \frac{1}{e^{7.4(\ln n)^{1 / 3}}} .
$$

Для описания доказательства теоремы введем обозначения. Пусть $0<\varepsilon<1$. Выберем натуральное $r$ так, чтобы выполнялось неравенство $10 / \varepsilon<r<12 / \varepsilon$. Положим $\delta_{1}=\varepsilon / 2, \delta_{2}=\varepsilon /(5 r), \delta_{3}=\varepsilon / 20$. Обозначим $h=n^{-1 / 2+\delta_{1}}, H=n^{1 / 2-\delta_{2}}$. Каждому набору целых чисел $\left(U_{0}, \ldots, U_{r-1}, V_{0}, \ldots, V_{r-1}\right)$ сопоставим $2 r$-мерньй куб

$$
\Pi=\Pi\left(U_{0}, \ldots, U_{r-1}, V_{0}, \ldots, V_{r-1}\right)=\prod_{\rho=0}^{r-1}\left(U_{\rho} h,\left(U_{\rho}+1\right) h\right] \times \prod_{\rho=0}^{r-1}\left(V_{\rho} h,\left(V_{\rho}+1\right) h\right] .
$$

Семейство $\Phi$ и множество $\Omega$ определяются как в работе [3]. Обозначим через $k_{0}$ наибольшее простое, не превосходящее $n^{1-\delta_{3}}$, и пусть $k_{1}=\left[k_{0} / 2\right]$.

Для целого $k$ и $x=x_{k}=2 \pi k / k_{0}$ рассмотрим событие $E_{k}$

$$
\begin{aligned}
& \left(\operatorname{Re} T(x), \operatorname{Re}\left(\frac{T^{\prime}(x)}{i n}\right), \ldots, \operatorname{Re}\left(\frac{T^{(r-1)}(x)}{(i n)^{r-1}}\right),\right. \\
& \left.\quad \operatorname{Im} T(x), \operatorname{Im}\left(\frac{T^{\prime}(x)}{i n}\right), \ldots, \operatorname{Im}\left(\frac{T^{(r-1)}(x)}{(i n)^{r-1}}\right)\right) \in \Omega .
\end{aligned}
$$

Обозначим через $G_{0}$ событие

$$
\max _{s \in T}\left|T^{(r)}(s)\right|<n^{r+2}
$$

Далее, будем считать, что $n>\left(16 C_{0}(\xi)\right)^{9936 / \varepsilon^{3}}$. Имеют место следуюшие утверждения.

ЛЕмма 1. Если при некотором $k, 0<k<k_{0} / 2$, произошло событие $E_{k} \cap G_{0}$, mo

$$
\min _{s \in\left[x_{k}-\Delta, x_{k}+\Delta\right]}|T(s)| \leqslant n^{-1 / 2+\varepsilon},
$$

əде $\Delta=n^{-1-\delta_{1}}$.

ЛЕмма 2. Имеет место неравенство

$$
\operatorname{Pr}\left(G_{0}\right)>1-\frac{1}{n^{3 / 2}}
$$

Лемма 3. Имеет место неравенство

$$
\operatorname{Pr}\left(\min _{s \in T}|T(s)|>n^{-1 / 2+\varepsilon}\right)<1-\operatorname{Pr}\left(\bigcup_{k=1}^{k_{1}} E_{k}\right)+\frac{1}{n^{3 / 2}}
$$


Лемма 4. Имеет место неравенство

$$
V_{\Omega} n^{-r} k_{0}>\frac{\pi^{r-1}}{88 \cdot 4^{r}} \cdot n^{\varepsilon / 20}
$$

где $V_{\Omega}-$ облем множества $\Omega$.

Рассмотрим случайные векторы $X_{j}, j=0, \ldots, n-1$, для $x=x_{k}=2 \pi k / k_{0}$, где $k-$ фиксированное число, $0<k<k_{0} / 2$,

$$
\begin{aligned}
X_{j}= & \xi_{j}\left(\cos (j x), \cos (j x)\left(\frac{j}{n}\right), \ldots, \cos (j x)\left(\frac{j}{n}\right)^{r-1},\right. \\
& \left.\sin (j x), \sin (j x)\left(\frac{j}{n}\right), \ldots, \sin (j x)\left(\frac{j}{n}\right)^{r-1}\right)
\end{aligned}
$$

и определим для них ковариационную матрицу [4, с. 72$]$

$$
V=\frac{1}{n} \sum_{j=0}^{n-1} \operatorname{Cov}\left(X_{j}\right)
$$

Обозначим через $f$ характеристическую функцию случайного вектора

$$
X=\sum_{j=0}^{n-1} X_{j}
$$

Разобьем $\mathbb{R}^{2 r}$ на три множества: $\mathbb{R}^{2 r}=(I) \cup(I I) \cup(I I I)$, где

$$
\begin{gathered}
(I)=\left\{\omega:\|\omega\| \leqslant n^{-1 / 2+1 / 7}\right\}, \quad(I I)=\left\{\omega: n^{-1 / 2+1 / 7}<\|\omega\| \leqslant n^{1 / 2}\right\}, \\
(I I I)=\left\{\omega:\|\omega\|>n^{1 / 2}\right\} .
\end{gathered}
$$

Для характеристической функции $f$ устанавливаются следующие оценки:

$$
\left|f(\omega)-\exp \left\{-\frac{n}{2}\langle\omega, V \omega\rangle\right\}\right| \leqslant \begin{cases}\frac{1}{n^{1 / 15}} \exp \left\{-\frac{n}{2}\langle\omega, V \omega\rangle\right\} & \text { при } \omega \in(I), \\ |f(\omega)| \leqslant \exp \left(-n^{1 / 7}\right) & \text { при } \omega \in(I I) .\end{cases}
$$

Для доказательства первой оценки используются теорема 8.4 [4], а также неравенства, полученные для произвольных многочленов $P$ и $Q$ степени $r-1$, заданных на отрезке $[0,1]$,

$$
\sum_{j=0}^{r-1}\left|a_{j}\right|^{2} \leqslant 9^{2 r} \int_{0}^{1} P^{2}(t) d t
$$

где $a_{0}, \ldots, a_{r-1}-$ коэффициенты $P$,

$$
\frac{1}{4}\left(\|P\|_{L_{2}}^{2}+\|Q\|_{L_{2}}^{2}\right) \leqslant \frac{1}{n} \sum_{j=0}^{n-1}\left[\cos (j x) P\left(\frac{j}{n}\right)+\sin (j x) Q\left(\frac{j}{n}\right)\right]^{2} \leqslant \frac{3}{4}\left(\|P\|_{L_{2}}^{2}+\|Q\|_{L_{2}}^{2}\right) .
$$

Далее, мы доказьваем лемму. 
ЛЕмма 5. Справедлива оценка

$$
\left|\int_{\mathbb{R}^{2 r}} \exp \left\{-\frac{n}{2}\langle\omega, V \omega\rangle\right\} d \omega-\frac{\gamma_{r}}{n^{r}}\right| \leqslant \frac{\gamma_{r}}{n^{r}} \cdot \frac{1}{n^{\delta_{3} / 2}},
$$

əде

$$
\gamma_{r}=\frac{(2 \pi)^{r}}{\left(\operatorname{det} V^{1}\right)^{1 / 2}}, \quad V^{1}=\left(\begin{array}{cc}
V^{0} & 0 \\
0 & V^{0}
\end{array}\right)
$$

$a V^{0}-$ матрича с әлементами

$$
v_{\rho, \rho^{\prime}}^{0}=\frac{\mathrm{M} \xi^{2}}{2\left(\rho+\rho^{\prime}+1\right)}, \quad 0 \leqslant \rho<r, \quad 0 \leqslant \rho^{\prime}<r .
$$

Положим для $t \in[0,1]$

$$
\varphi(t)=-\int_{0}^{t} x^{2 r^{2}}(1-x)^{2 r^{2}} d x / \int_{0}^{1} x^{2 r^{2}}(1-x)^{2 r^{2}} d x+1
$$

и

$$
\psi(t)= \begin{cases}\varphi(t) & \text { на }[0,1], \\ \varphi(-t) & \text { на }[-1,0), \\ 0 & \text { на }(-\infty,-1) \cup(1, \infty) .\end{cases}
$$

Тогда $\psi \in C^{2 r^{2}}(-\infty, \infty)$ - четная неотрицательная функция с носителем на $[-1,1]$, не превосходящая 1, с единичньм интегралом. Далее, рассматриваем $2 r$-мерный куб П с ребрами, параллельными координатньм осям и равньми $h$, содержащийся в кубе $[-H, H]^{2 r}$, и куб $\Pi_{1}-$ образ П при гомотетии с центром в центре П и коэффициентом $\left(n^{\delta_{2}}+1\right) / n^{\delta_{2}}$. По аналогии с [3] с помощью $\psi$ определяются $\psi_{\Pi}, \Psi_{\Pi}, F_{\Pi}, \psi_{\Pi_{1}}, \Psi_{\Pi_{1}}, F_{\Pi_{1}}$. Далее, устанавливаем неравенство

$$
\frac{1}{(2 \pi)^{2 r}} \cdot \frac{1}{\Psi_{\Pi}} \int_{\mathbb{R}^{2 r}} f(\omega) F_{\Pi}(-\omega) d \omega \leqslant \operatorname{Pr}(X \in \Pi) \leqslant \frac{1}{(2 \pi)^{2 r}} \cdot \frac{1}{\Psi_{\Pi_{1}}} \int_{\mathbb{R}^{2 r}} f(\omega) F_{\Pi_{1}}(-\omega) d \omega,
$$

а с помощью оценок для функции $f$ и леммы 5 неравенства

$$
\begin{aligned}
\left|\int_{\mathbb{R}^{2 r}} f(\omega) F_{\Pi}(-\omega) d \omega-\frac{\gamma_{r}}{n^{r}}\right| & \leqslant \frac{\gamma_{r}}{n^{r}} \cdot \frac{1}{n^{2 \delta_{2} / 3}}, \\
\left|\int_{\mathbb{R}^{2 r}} f(\omega) F_{\Pi_{1}}(-\omega) d \omega-\frac{\gamma_{r}}{n^{r}}\right| & \leqslant \frac{\gamma_{r}}{n^{r}} \cdot \frac{1}{n^{2 \delta_{2} / 3}},
\end{aligned}
$$

с помошью которых получаем неравенство

$$
\left|\operatorname{Pr}(X \in \Pi)-\frac{\gamma_{r}}{n^{r}} \cdot \frac{V_{\Pi}}{(2 \pi)^{2 r}}\right| \leqslant \frac{\gamma_{r}}{n^{r}} \cdot \frac{V_{\Pi}}{(2 \pi)^{2 r}} \cdot \frac{1}{n^{\delta_{2} / 2}} \quad\left(V_{\Pi}=h^{2 r}\right),
$$

из которого выводятся оценки для вероятностей событий $E_{k}, 1 \leqslant k \leqslant k_{1}$,

$$
\left|\operatorname{Pr}\left(E_{k}\right)-\Gamma\right| \leqslant \frac{\Gamma}{n^{\delta_{2} / 2}},
$$


где

$$
\Gamma=\frac{\gamma_{r}}{(2 \pi)^{2 r}} \cdot \frac{V_{\Omega}}{n^{r}}
$$

Рассматривая попарные объединения $Y$ векторов $X$ [3], мы устанавливаем оценки для характеристических функций векторов $Y$, с помощњю которых получаем неравенство

$$
\left|\operatorname{Pr}\left(Y \in \Pi \times \Pi^{\prime}\right)-\left(\frac{\gamma_{r}}{n^{r}} \cdot \frac{V_{\Pi}}{(2 \pi)^{2 r}}\right)^{2}\right| \leqslant\left(\frac{\gamma_{r}}{n^{r}} \cdot \frac{V_{\Pi}}{(2 \pi)^{2 r}}\right)^{2} \cdot \frac{1}{n^{\delta_{2} / 2}},
$$

где П, П' - $2 r$-мерные кубы с ребрами, параллельньми координатным осям и равными $h$, содержащиеся в кубе $[-H, H]^{2 r}$. Отсюда получаются оценки для вероятностей попарных пересечений $E_{i} \cap E_{j}, 1 \leqslant i<j \leqslant k_{1}$,

$$
\left|\operatorname{Pr}\left(E_{i} \cap E_{j}\right)-\Gamma^{2}\right| \leqslant \Gamma^{2} \frac{1}{n^{\delta_{2} / 2}}
$$

С помощью оценок $\operatorname{Pr}\left(E_{k}\right)$ и $\operatorname{Pr}\left(E_{i} \cap E_{j}\right)$, используя метод нормального порядка [3], мы устанавливаем соотношение

$$
1-\operatorname{Pr}\left(\bigcup_{k=1}^{k_{1}} E_{k}\right) \leqslant \frac{14}{n^{\delta_{2} / 2}}
$$

откуда с помощью леммы 3 завершаем доказательство теоремы.

Автор благодарит С. В. Конягина за полезные обсуждения и ценные рекомендации.

Московский государственный университет

Поступило

им. М. В. Ломоносова

10.11 .96

\section{СПИСОК ЦИТИРОВАННОЙ ЛИТЕРАТУРЫ}

1. Littlewood J. E. // J. London Math. Soc. 1966. V. 41. P. 367-376. 2. Кашин Б. С. // Вестн. МГУ. Сер. 1. Матем., мех. 1987. № 5. С. 40-46. 3. Конягин С. В. // Матем. заметки. 1994. Т. 56. № 3. С. 80-101. 4. Бхаттачария Р. Н., Ранга Рао Р. Аппроксимация нормальньм распределением и асимптотические разложения. М.: Наука, 1982. 